\title{
Antimicrobial Use at Acute Care Hospitals in Nova Scotia: A Point Prevalence Survey
}

\author{
Emily Black, Heather Neville, Mia Losier, Megan Harrison, Kim Abbass, Kathy Slayter, Lynn Johnston, \\ and Ingrid Sketris
}

\begin{abstract}
Background: Point prevalence surveys are used to monitor antimicrobial use and identify targets for improvement through antimicrobial stewardship activities. Few studies have evaluated antimicrobial use in Nova Scotia acute care institutions.

Objectives: To determine the prevalence and characteristics of antimicrobial use in Nova Scotia hospitals.

Methods: A point prevalence survey was conducted between June and November 2015 for patients admitted to hospitals with at least 30 acute care beds. On each survey day, charts were reviewed to identify patients receiving antimicrobial agents on that day. Data were gathered on the type of antimicrobial agent prescribed, route of administration, intended duration of use, and indication. Adherence to regional and local treatment guidelines was assessed. Results were summarized descriptively. Findings were compared using the Fisher exact test or the Cochran-Armitage trend test.
\end{abstract}

Results: Twelve of the 13 eligible hospitals participated, and a total of 1499 patient charts were examined. The overall prevalence of antimicrobial use was 30.6\% (458/1499). The prevalence of antimicrobial use differed significantly according to area of specialty, with the highest prevalence occurring in intensive care wards $(47.2 \%, 50 / 106)$ and surgical wards $(43.4 \%, 179 / 412)$, as compared with medical wards $(27.9 \%, 192 / 687)$ and "other" specialty wards $(11.1 \%, 32 / 289)(p<0.001)$. Among the 520 indications for antimicrobial use, the most common was respiratory tract infection (81 or $15.6 \%$ ). In total, 660 antimicrobial agents were prescribed to the 458 patients; a third of these patients (152 or 33.2\%) received more than 1 antimicrobial agent. The class of antimicrobials most frequently prescribed was "other beta-lactam antimicrobials" $(31.2 \%$, 206/660). The majority of antimicrobials (62.0\%, 409/660) were prescribed for administration via the parenteral route. Adherence to regional treatment guidelines was $29.9 \%$ (26 of 87 indications analyzed). Documentation of indication was lacking for 104 (20.0\%) of the 520 indications, and documentation of the intended duration of antimicrobial use was lacking for 326 (62.7\%) of the 520 indications.

Conclusions: Antimicrobial agents were prescribed for about one-third of acute care patients in Nova Scotia. Specific targets for improvement in antimicrobial use include decreases in prescribing of broad-spectrum and parenteral antimicrobials, better adherence to guidelines, and improved documentation. In developing initiatives, antimicrobial stewardship programs in Nova Scotia should focus on identified targets for improvement.

Keywords: antimicrobial utilization, antimicrobial stewardship, antimicrobial agent, antibiotic

\section{RÉSUMÉ}

Contexte: Les enquêtes de prévalence ponctuelle sont employées pour surveiller l'utilisation des antimicrobiens et cibler des points à améliorer grâce aux activités de gestion responsable des antimicrobiens. Peu d'études se sont penchées sur l'utilisation des antimicrobiens dans les établissements de soins de courte durée en Nouvelle-Écosse.

Objectifs : Déterminer quelle est la prévalence de l'utilisation des antimicrobiens dans les hôpitaux de la Nouvelle-Écosse et offrir un portrait de cette utilisation.

Méthodes : Une enquête de prévalence ponctuelle a été menée entre juin et novembre 2015 pour les patients admis aux hôpitaux dotés d'au moins 30 lits de soins de courte durée. À chaque jour d'enquête, des dossiers médicaux ont été examinés afin de repérer les patients ayant reçu des agents antimicrobiens cette journée-là. On a recueilli des données sur le type d'agent antimicrobien prescrit, la voie d'administration, la durée attendue d'utilisation et l'indication. Le respect des lignes directrices thérapeutiques régionales et locales a aussi été évalué. Les résultats ont été résumés de façon descriptive. Les comparaisons ont été vérifiées à l'aide du test exact de Fisher ou du test de tendance de Cochran-Armitage.

Résultats : Douze des 13 hôpitaux admissibles ont été inclus et un total de 1499 dossiers médicaux de patients ont été examinés. Le taux de prévalence globale d'utilisation d'antimicrobiens était de 30,6\% (458/1499). La prévalence d'utilisation d'antimicrobiens variait significativement selon les unités de soins : en tête de liste, les unités de soins intensifs (47,2\%, $50 / 106)$ et les unités de chirurgie $(43,4 \%, 179 / 412)$ comparativement aux unités de médecine $(27,9 \%, 192 / 687)$ et aux " autres " unités de soins $(11,1 \%, 32 / 289)(p<0.001)$. Parmi les 520 indications pour l'utilisation des antimicrobiens, la plus fréquente était l'infection des voies respiratoires (81 ou 15,6\%). Au total, 660 agents antimicrobiens ont été prescrits aux 458 patients et le tiers de ces patients (152 ou 33,2\%) ont reçu plus d'un agent antimicrobien. La classe d'antimicrobien la plus souvent prescrite était les " autres bêtalactamines " $(31,2 \%, 206 / 660)$. La voie parentérale était prescrite pour l'administration de la majorité des antimicrobiens $(62,0 \%, 409 / 660)$. Le respect des lignes directrices régionales de traitement était de 29,9\% (26 des 87 indications analysées). Parmi les 520 indications, 104 (20,0\%) n'étaient pas mentionnées au dossier et $326(62,7 \%)$ étaient dépourvues de mention de la durée du traitement antimicrobien au dossier.

Conclusions : Des agents antimicrobiens ont été prescrits à environ un tiers des patients recevant des soins de courte durée en Nouvelle-Écosse. Lamélioration de l'utilisation des antimicrobiens devrait cibler précisément les réductions de la prescription d'antibiotiques à large spectre et du 


\section{Can J Hosp Pharm. 2018;71(4):234-42}

recours à la voie parentérale, un plus grand respect des lignes directrices et une meilleure consignation. Les programmes de gestion responsable des antimicrobiens en Nouvelle-Écosse devraient être axés sur des objectifs d'amélioration définis afin de mettre au point des stratégies.

Mots clés : utilisation des antimicrobiens, gestion responsable des antimicrobiens, agent antimicrobien, antibiotiquee

\section{INTRODUCTION}

$\mathrm{I}^{\mathrm{n}}$ nternational surveillance has shown that antimicrobial resistance is increasing. ${ }^{1,2}$ In 2016, the Director-General of the World Health Organization (WHO) warned that "antimicrobial resistance poses a fundamental threat to human health, development, and security". ${ }^{3}$ Inappropriate antimicrobial use, occurring in more than one-quarter of all antimicrobial courses prescribed, ${ }^{4}$ increases the risk of resistance. In addition, antimicrobial resistance leads to negative health consequences, including a statistically significant increase in mortality. ${ }^{5}$

Because of antimicrobial resistance, Canadians have been encouraged to work collaboratively to identify solutions through surveillance, stewardship, infection prevention and control, and innovation. ${ }^{6}$ Several strategies to reduce the risk of resistance can be considered. ${ }^{7,8}$ However, to identify solutions and tailor strategies to improve antimicrobial use, an evaluation of antimicrobial utilization is needed.

Point prevalence surveys are a suggested strategy to evaluate antimicrobial use. They have been used nationally and internationally to determine the prevalence of antimicrobial use and to identify areas for quality improvement. ${ }^{9-18}$ According to previously published transnational and Canadian point prevalence surveys of antimicrobial use, about one-third of patients admitted to hospital are receiving antimicrobial agents at any given time. ${ }^{12,13,15,16}$

Recent statistics on antimicrobial use based on individual patient-level prescribing data at acute care hospitals in Nova Scotia have not been published. A study that utilized purchasing data for this province suggested an increase in use of fluoroquinolones, from 47.2 defined daily doses (DDD) per 1000 bed-days per year in $1997 / 98$ to $163.8 \mathrm{DDD} / 1000$ bed-days per year in 2002/03. ${ }^{19}$ In addition, the Canadian Antimicrobial Resistance Surveillance System Report 2016 highlighted that in 2014, Nova Scotia had the second highest number of DDD per patient discharge for antimicrobials purchased by hospitals across the country. ${ }^{20} \mathrm{~A}$ current assessment of antimicrobial prescribing in Nova Scotia, with consideration of regional variation in utilization, prescribing indication, and adherence to guidelines, was therefore needed.

The purpose of this study was to determine the prevalence of antimicrobial use in Nova Scotia hospitals; to characterize antimicrobial use in terms of drug selection, route of administration, and indication for prescribing; to compare antimicrobial use by population size, age, and area of specialty; to determine adherence to regional and local guidelines (where they exist); and to determine targets for quality improvement in antimicrobial use.

\section{METHODS}

This study was a point prevalence survey of antimicrobial use by patients admitted to acute care hospitals in Nova Scotia, Canada. This study was approved by the research ethics boards of the Nova Scotia Health Authority on April 15, 2015 (File No. 100287), and the IWK Health Centre on September 8, 2015 (File No. 1020269). Both research ethics boards waived the need for informed consent.

\section{Study Setting and Patient Population}

This study was completed in Nova Scotia. At the time of this study, Nova Scotia had a population of $941545 .{ }^{21}$ In 2015 , just before data collection, 9 health authorities in the province were merged into a single health authority (the Nova Scotia Health Authority), in addition to the IWK Health Centre. The Nova Scotia Health Authority has 1 tertiary specialty hospital (the Queen Elizabeth II Health Sciences Centre), 8 regional hospitals, and about 135 other facilities. ${ }^{22}$ In addition to hospitals in the Nova Scotia Health Authority, the province has 1 specialized hospital (the IWK Health Centre), which provides primary, secondary, and tertiary care to women, children, youth, and families. $^{23}$

All hospitals in Nova Scotia with at least 30 inpatient acute care beds at the time of the survey were invited to participate. Smaller hospitals were excluded because available funding was insufficient to collect data at all hospitals throughout the province. Participating hospitals were asked to complete a questionnaire summarizing institution characteristics (hospital type, number of beds) and on-site availability of antimicrobial stewardship or infectious disease expertise. Teaching hospitals were defined as institutions providing highly complex patient care, having a formal partnership with a medical or health sciences school, and having substantial research activity and postgraduate training. ${ }^{24,25}$ All other hospitals were defined as community hospitals. Although these institutions constituted a single health authority (excluding the IWK Health Centre) at the time of data collection, differences in formulary restrictions and antimicrobial policies existed throughout the province. 
Within each participating hospital, all patients who had been admitted to an acute care bed for at least $24 \mathrm{~h}$ at 0800 on the particular institution's survey date(s) were screened for eligibility. Admitted patients in the emergency department, long-term care, restorative care, transitional care, and rehabilitation beds were excluded. Among eligible patients, those for whom a systemic antimicrobial agent had been prescribed were identified and included in the survey.

\section{Data Collection}

Data on antimicrobial use were collected by members of the research team over the period June 22 to November 2, 2015. On each survey day, a census of admitted patients was electronically generated for a particular hospital ward. Pharmacists, pharmacy students (who had completed second or third year), and a pharmacy technician used the electronic patient census to identify patients and collect data from paper-based charts. As a quality control measure, to ensure accuracy of data collection, data extraction was assessed by a second individual for $10 \%$ of the charts at each site. Any discrepancies in data collection were identified, discussed, and resolved the same day. All eligible patients admitted to the same hospital ward within a given institution were surveyed on the same day. All acute care hospital wards within a participating institution were surveyed within the same 3- to 4-week period.

The European Surveillance of Antimicrobial Consumption Network (ESAC-Net) is a European surveillance network that has developed a standardized methodology for assessing antimicrobial utilization through point prevalence surveys. With permission, a standardized data collection form for the current study was developed on the basis of the network's 2009 form. The European Centre for Disease Prevention and Control (ECDC) has adopted the point prevalence survey methodology of ESAC-Net to monitor antimicrobial use and has published a technical document that provided guidance in completing data collection. ${ }^{26}$

Information was gathered on the type of clinical ward, patient demographic characteristics, antimicrobial utilization, and indication for prescribing. Information collected on the clinical ward included the name of the ward, area of specialty (if applicable), and total number of patients who had been admitted to the ward for at least $24 \mathrm{~h}$ at 0800 on the day of the survey. The type of antimicrobial agent was coded according to the WHO's classification index for the Anatomical Therapeutic Chemical (ATC) system, 2015 edition. ${ }^{27}$ Included systemic antimicrobials were antibacterials, antimycotics, antimycobacterials, antivirals, intestinal anti-infectives, and antiprotozoals. Information collected on indication for antimicrobial prescribing was based on anatomic site.

\section{Guideline Adherence}

Data collectors (pharmacists and pharmacy students) assessed antimicrobial use against existing regional and locally approved evidence-based guidelines. Adherence to guidelines for antimicrobial selection, dose, and duration was assessed for communityacquired pneumonia, acute exacerbation of chronic obstructive pulmonary disease (COPD), urinary tract infections (UTIs), and nonpurulent cellulitis. Adherence to guidelines for surgical site prophylaxis was assessed at the IWK Health Centre. These indications were selected on the basis of availability of regional guidelines and their frequency of occurrence in this patient population. The Capital Health Antimicrobial Handbook $2012,{ }^{28}$ which is the source of the regionally developed guidelines for community-acquired pneumonia, UTIs, and cellulitis, was used to assess adherence to guidelines in the adult population at all hospitals within the Nova Scotia Health Authority. Adherence to guidelines in a regional preprinted order for management of acute exacerbation of COPD, developed by the Queen Elizabeth II Health Sciences Centre, was also assessed. In addition, adherence to local guidelines developed between 2008 and 2015 by individual community hospitals for the specified indications (where available) and the IWK Health Centre was evaluated. In the event that a data collector was uncertain about adherence to guidelines, a member of the research team was consulted. If adherence remained unclear, 2 additional members of the research team (infectious disease physician and/or clinical pharmacist) independently reviewed the case and provided a recommendation. If disagreement occurred at this stage, the case was reviewed by a third member of the research team (infectious disease physician or clinical pharmacist), whose recommendation prevailed.

\section{Data Analysis}

Prevalence and type of antimicrobial agent used, route of administration, indication for antimicrobial prescribing, documentation, and adherence to guidelines were summarized descriptively. Prevalence of antimicrobial use was reported at the patient level. Type of antimicrobial used, route of administration, and documentation of intended duration were reported at the prescription level. Indication for antimicrobial use, adherence to guidelines, and documentation of indication were reported as proportions of total indications in the study population. In calculating prevalence, the number of acute care patients admitted for at least $24 \mathrm{~h}$ and having an active prescription for 1 or more systemic antimicrobial agents at 0800 on the day of the survey represented the numerator, and the number of acute care patients admitted for at least 24 hours at 0800 on the day of the survey represented the denominator. 9 Findings for prevalence and antimicrobial use by age (17-65 years, $>65$ years), population size (small versus large population centre), and area of specialty (medicine versus surgery) were compared using the Fisher exact test. Findings for route of administration (oral, parenteral, inhalation) by population size (small versus large population centre) and age ( $0-16$ years, $17-65$ years, $>65$ years of age) were compared using the Cochran-Armitage trend test. The analysis did not control for type I error. 


\section{RESULTS}

\section{Hospital Characteristics}

Twelve of 13 hospitals meeting the inclusion criteria participated in the survey. Two of the hospitals were categorized as teaching hospitals located in large population centres (population $\geq 100000$ ), and the other 10 hospitals were community hospitals located in small or medium population centres (population between 1000 and 99 999). ${ }^{29}$ Both of the teaching hospitals employed infectious disease physicians certified by the Royal College of Physicians and Surgeons of Canada and dedicated infectious disease or antimicrobial stewardship pharmacists. Only 1 community hospital had a certified infectious disease physician, and another had an antimicrobial stewardship pharmacist; however, many of the community hospitals indicated that they were able to interact in a timely manner, via telephone consult, with an infectious disease physician from a larger teaching hospital or regional community hospital. At the time of data collection, antimicrobial stewardship committees had been established at 7 of the 12 participating study sites. Of the 5 sites that did not have stand-alone antimicrobial stewardship committees, 3 had an antimicrobial agent committee that reviewed stewardship policies.

\section{Antimicrobial Use}

The charts for a total of 1499 eligible hospital inpatients were reviewed; for 458 (30.6\%) of these patients, at least 1 antimicrobial agent was prescribed. Approximately one-third of the patients who were taking antimicrobial agents $(33.2 \%, 152 / 458)$ were receiving combination therapy. The baseline characteristics of the patient population are summarized in Table 1. A total of 660 antimicrobial agents were prescribed for 520 indications. The most common indications were respiratory tract infections
Table 1. Baseline Characteristics of Patients Receiving Antimicrobial Agents at Acute Care Hospitals in Nova Scotia

\begin{tabular}{lrc} 
Characteristic & No. $\begin{array}{c}\text { (\%) of Patients } \\
(\boldsymbol{n}=\mathbf{4 5 8})\end{array}$ \\
\hline Sex & & \\
Male & 221 & $(48.2)$ \\
Female & 233 & $(50.9)$ \\
Unknown & 4 & $(0.9)$ \\
\hline Age & & \\
$\leq 16$ years & 34 & $(7.4)$ \\
$17-65$ years & 193 & $(42.1)$ \\
$>65$ years & 229 & $(50.0)$ \\
Unknown & 2 & $(0.4)$ \\
\hline Type of hospital & & \\
Teaching & 225 & $(49.1)$ \\
Small or community & 233 & $(50.9)$ \\
\hline Specialty & & \\
Medical & 192 & $(41.9)$ \\
Surgical & 179 & $(39.1)$ \\
Intensive care & 50 & $(10.9)$ \\
Other & 32 & $(7.0)$ \\
Unknown & 5 & $(1.1)$ \\
\hline
\end{tabular}

$(15.6 \%, 81 / 520)$, prophylaxis for surgical site infections $(12.7 \%$, $66 / 520)$, and UTIs $(10.8 \%, 56 / 520)$. Indications for antimicrobial prescribing are summarized in Figure 1.

The prevalence of antimicrobial use differed significantly according to area of specialty, with the highest prevalence occurring in intensive care wards ( $47.2 \%$ [50 of 106 intensive care patients had at least 1 antimicrobial prescription]) and surgical wards $(43.4 \%, 179 / 412)$, as compared with medical wards

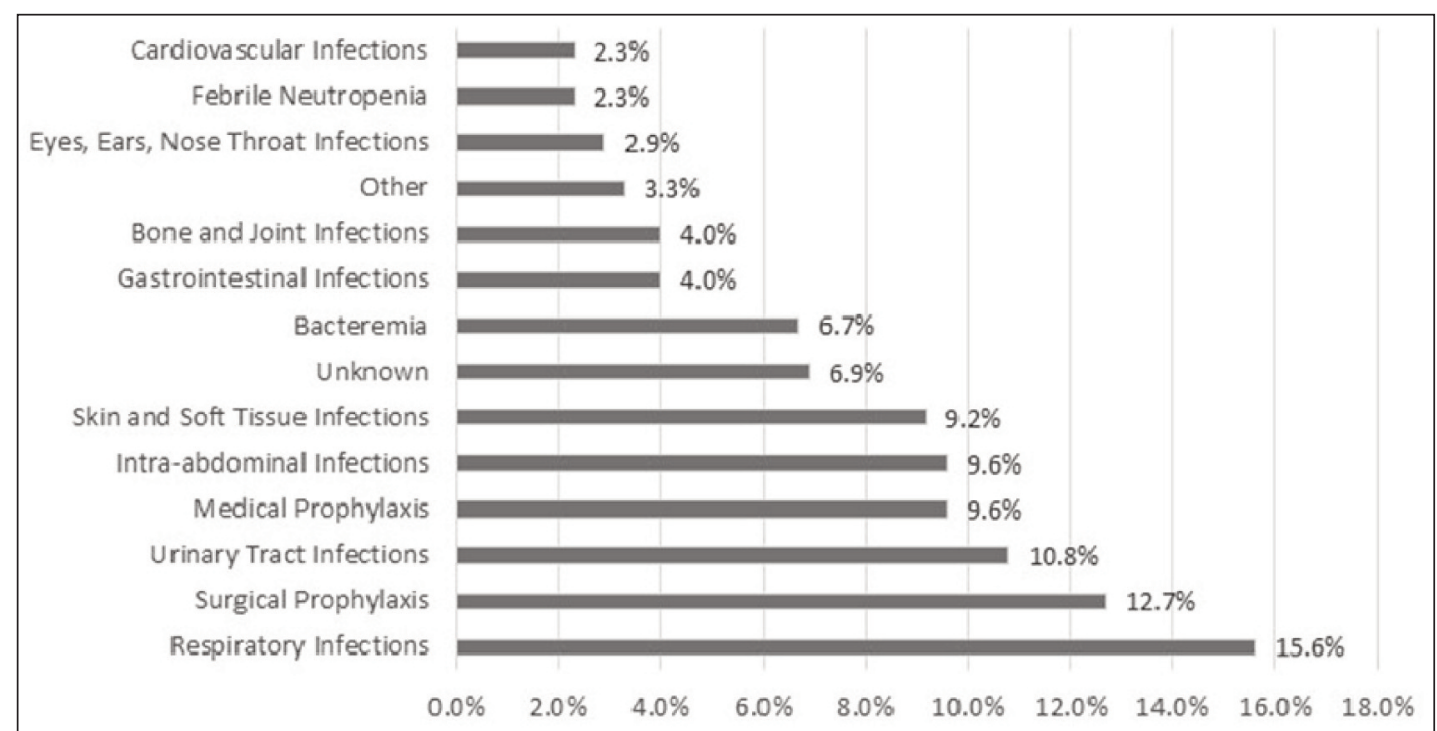

Figure 1. Indications for antimicrobial use at acute care hospitals in Nova Scotia $(n=520)$. 
$(27.9 \%, 192 / 687)$ and "other" specialty wards (11.1\%, 32/289) $(p<0.001)$. The other specialty wards were obstetrics/gynecology, pediatrics, family/newborn, palliative care, geriatrics, and mental health; area of specialty was unknown for 5 patients. Prevalence throughout the province, by hospital, ranged from $22.6 \%$ (53/234) to $43.5 \%$ (30/69). Prevalence of antimicrobial use at the community hospitals in small to medium population centres was significantly lower than at the teaching hospitals in large population centres $(27.6 \%$ [233/845] versus $34.4 \%$ [225/654]; $p=0.005)$.

Overall, 62.0\% (409/660) of the prescriptions were for parenteral antimicrobial agents. There was a statistically significant trend for younger patients to receive parenteral antimicrobials more often than older patients $(p<0.001)$. Parenteral administration was highest in the pediatric population up to 16 years of age $(82.8 \%$, or 48 of the 58 prescriptions for patients in this age group) and was lower in adults aged 17-65 years (65.7\%, $197 / 300)$ and those $>65$ years of age $(54.3 \%, 163 / 300)$; data on route of administration by age were missing for 2 antimicrobial orders. There was no statistically significant difference in rate of parenteral administration between large and small population centres $(62.9 \%, 217 / 345$, versus $60.95 \%, 192 / 315 ; p=0.08)$.

Antimicrobial use by drug class, based on the third level (pharmacologic subgroup) of the WHO ATC classification system, 2015 edition, ${ }^{27}$ is summarized in Figure 2. The most common class of antimicrobial agents prescribed was "other betalactam antibacterials" (31.2\%, 206/660), with cephalosporins representing the majority of antimicrobial agents $(27.6 \%$, $182 / 660)$ prescribed in this category. The most frequently prescribed antimicrobial agents at acute care hospitals in Nova
Scotia were metronidazole $(11.1 \%, 73 / 660)$, cefazolin $(10.9 \%$, $72 / 660)$, and ceftriaxone $(8.9 \%, 59 / 660)$. Antimicrobial use was similar by population size, with the exception of ciprofloxacin, which was prescribed more often in small to medium population centres than in large population centres $(11.1 \%$ [35/315] versus $4.1 \%$ [14/345]; $p<0.001)$, and cefazolin, which was prescribed more often in large population centres than in small to medium population centres (13.3\% [46/345] versus $8.3 \%$ [26/315]; $p=0.045)$. The drugs prescribed most commonly, overall and by population size, are shown in Figure 3.

Antimicrobial use by age for adult patients at acute care hospitals in Nova Scotia was also summarized, with similar utilization rates in patients aged 17 to 65 years and those older than 65 years, with the exception of ciprofloxacin, which was prescribed more often for those older than 65 years $(4.7 \%$ [14/300] versus $11.3 \%$ [34/300]) and piperacillin-tazobactam which was prescribed more often for those 17-65 years of age (10.7\% [32/300] versus 6.0\% [18/300]) (Figure 4). A greater proportion of patients admitted for surgery than for medical reasons had a prescription for cefazolin. Otherwise, the most commonly prescribed antimicrobial agents were comparable between specialties (Figure 5).

\section{Adherence to Regional and Local Guidelines}

Adherence to regional guidelines was assessed in relation to indication for 87 cases (involving community-acquired pneumonia, acute exacerbation of COPD, UTIs, or nonpurulent cellulitis). Adherence was unclear in 9 cases, which were sent for further independent review by 2 members of the research team. One case

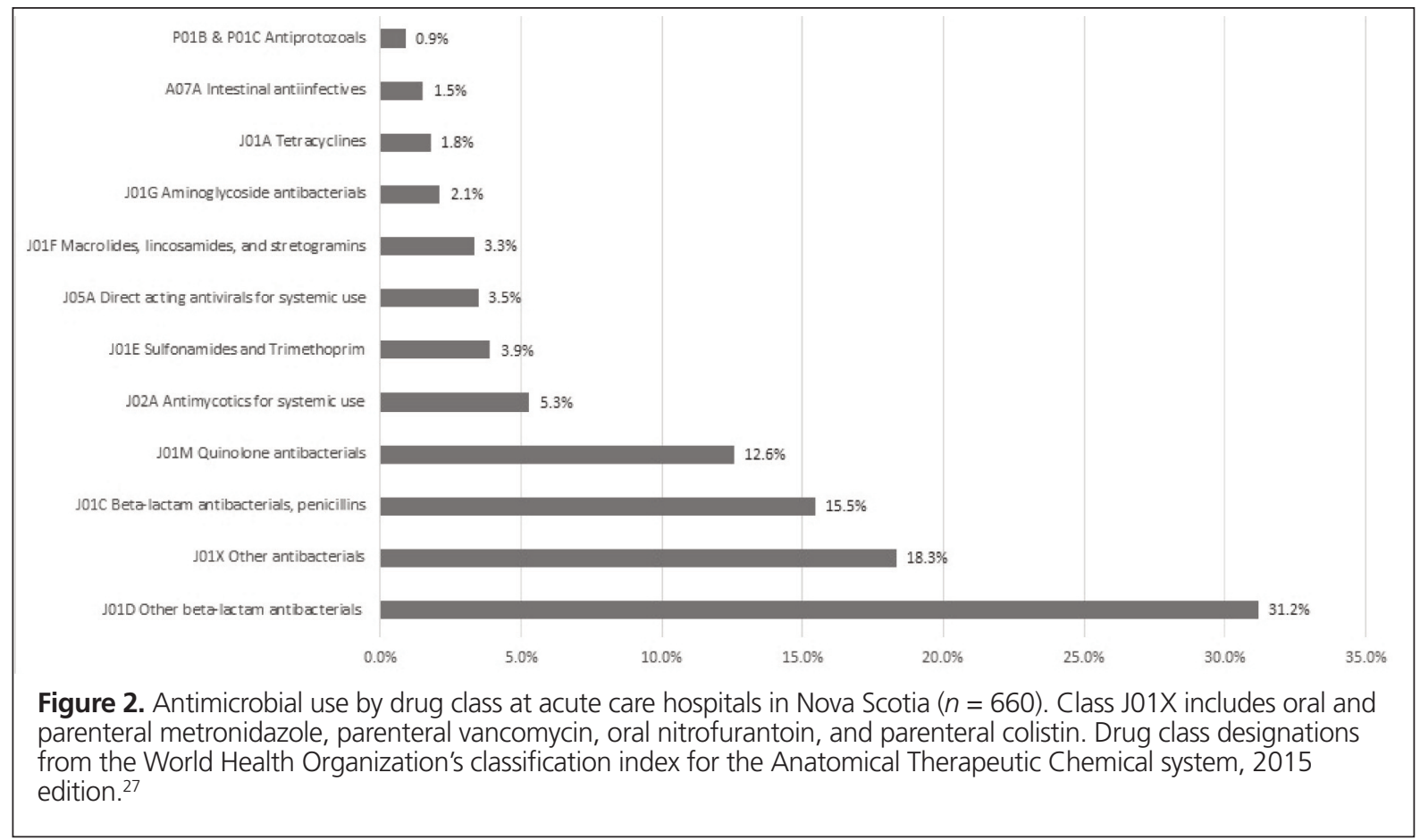




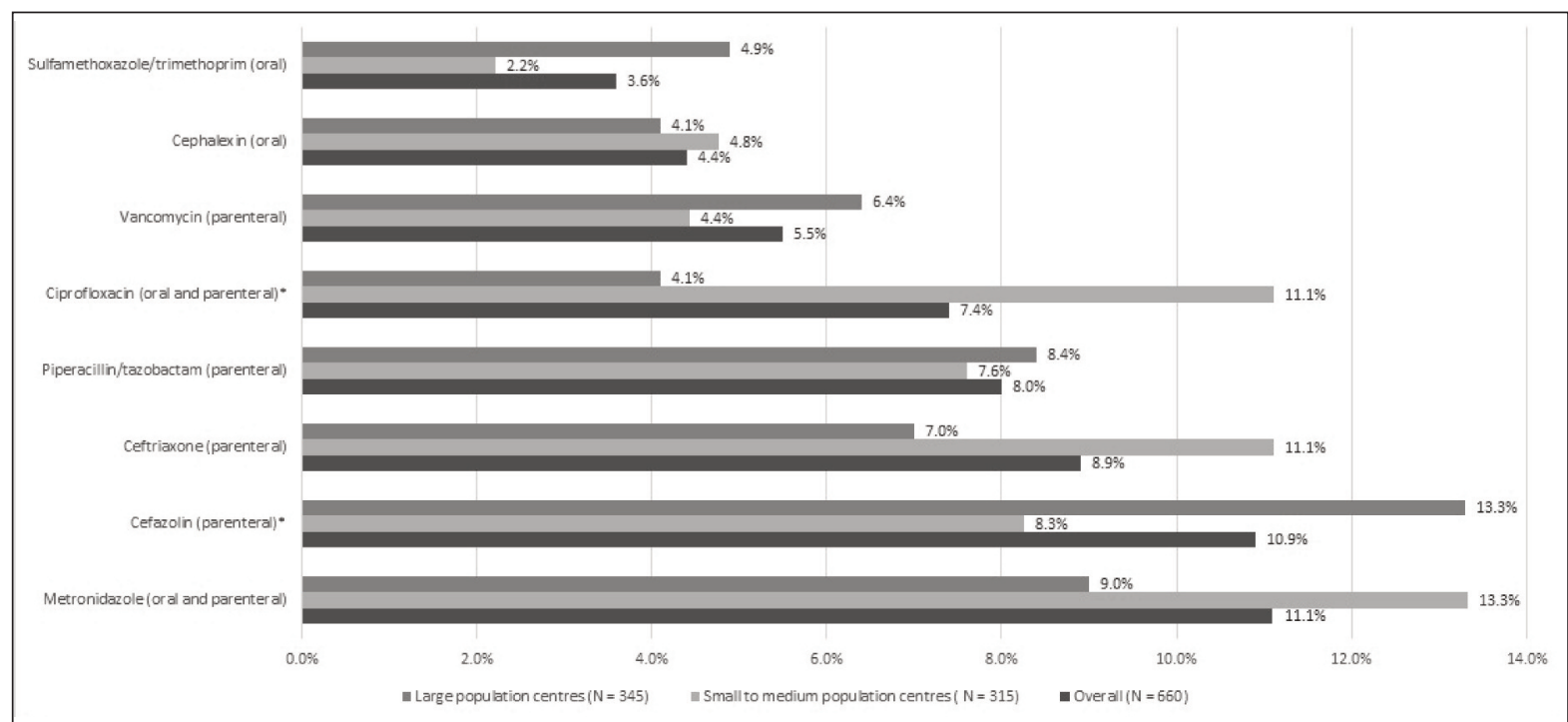

Figure 3. Antimicrobial agents prescribed most frequently at acute care hospitals in Nova Scotia. ${ }^{\star} p<0.05$.

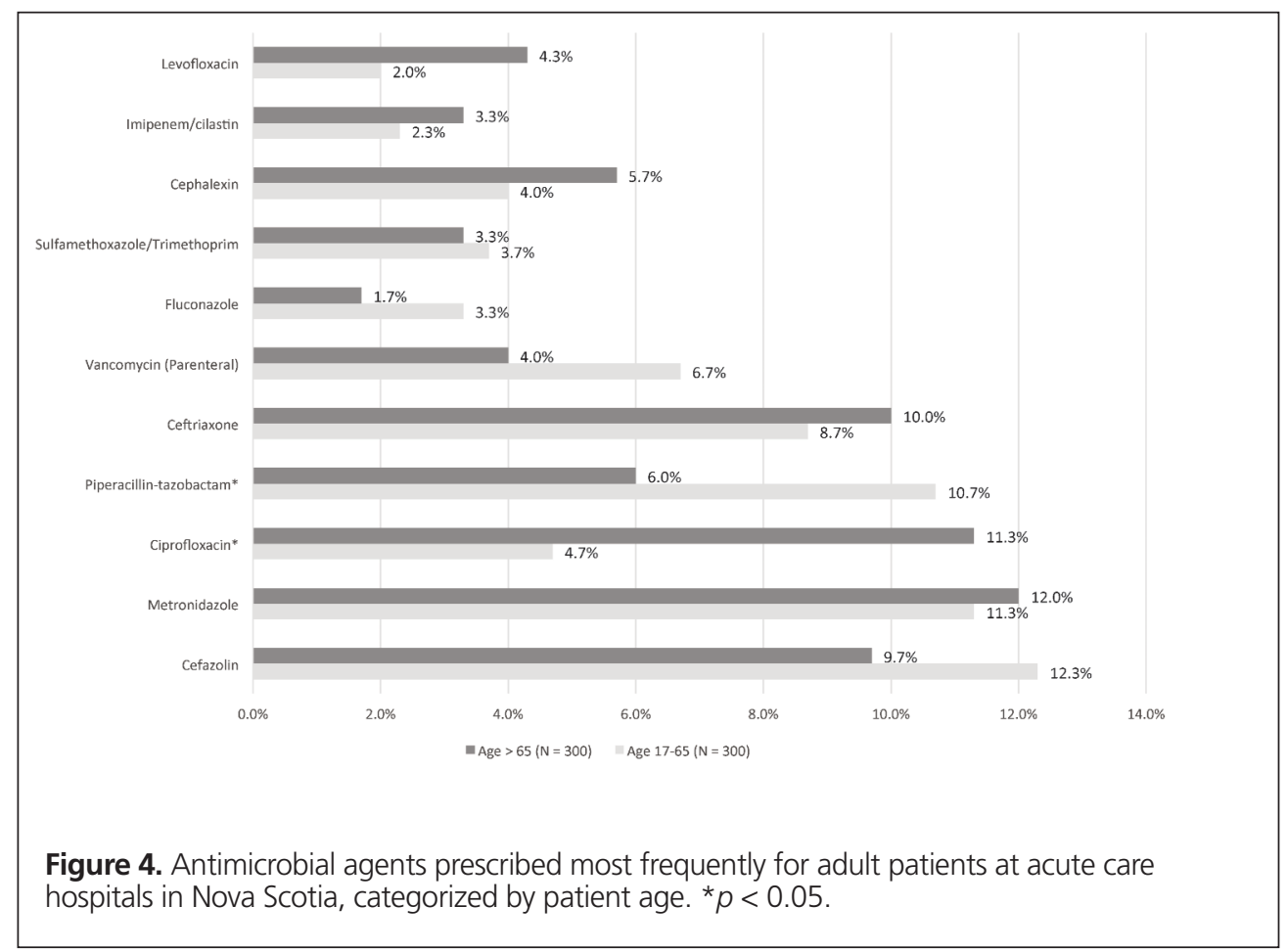

resulted in disagreement that required consideration by a third reviewer. Prescribers were adherent to regionally developed guidelines in $30 \%(26 / 87)$ of the cases assessed. The most common reason for nonadherence to regional guidelines was use of a second-line agent without a compelling reason to avoid the first-line antimicrobial (51\% [31 of 61 cases with nonadherence]). Regional UTI guidelines were most commonly evaluated, with prescribers adhering to guidelines in $31 \%(16 / 51)$ of cases. Adherence to regional guidelines was $29 \%$ (5/17) for communityacquired pneumonia and 45\% (5/11) for nonpurulent cellulitis.
Adherence to guidelines for acute exacerbation of COPD cannot be reported here because of small cell size (as per ethics requirements). Adherence to locally developed guidelines at community hospitals was 77\% (17/22) and at the IWK Health Centre was $71 \%(5 / 7)$.

\section{Documentation}

Documentation of the indication for antimicrobial prescribing occurred for $80.0 \%(416 / 520)$ of indications. Intended 


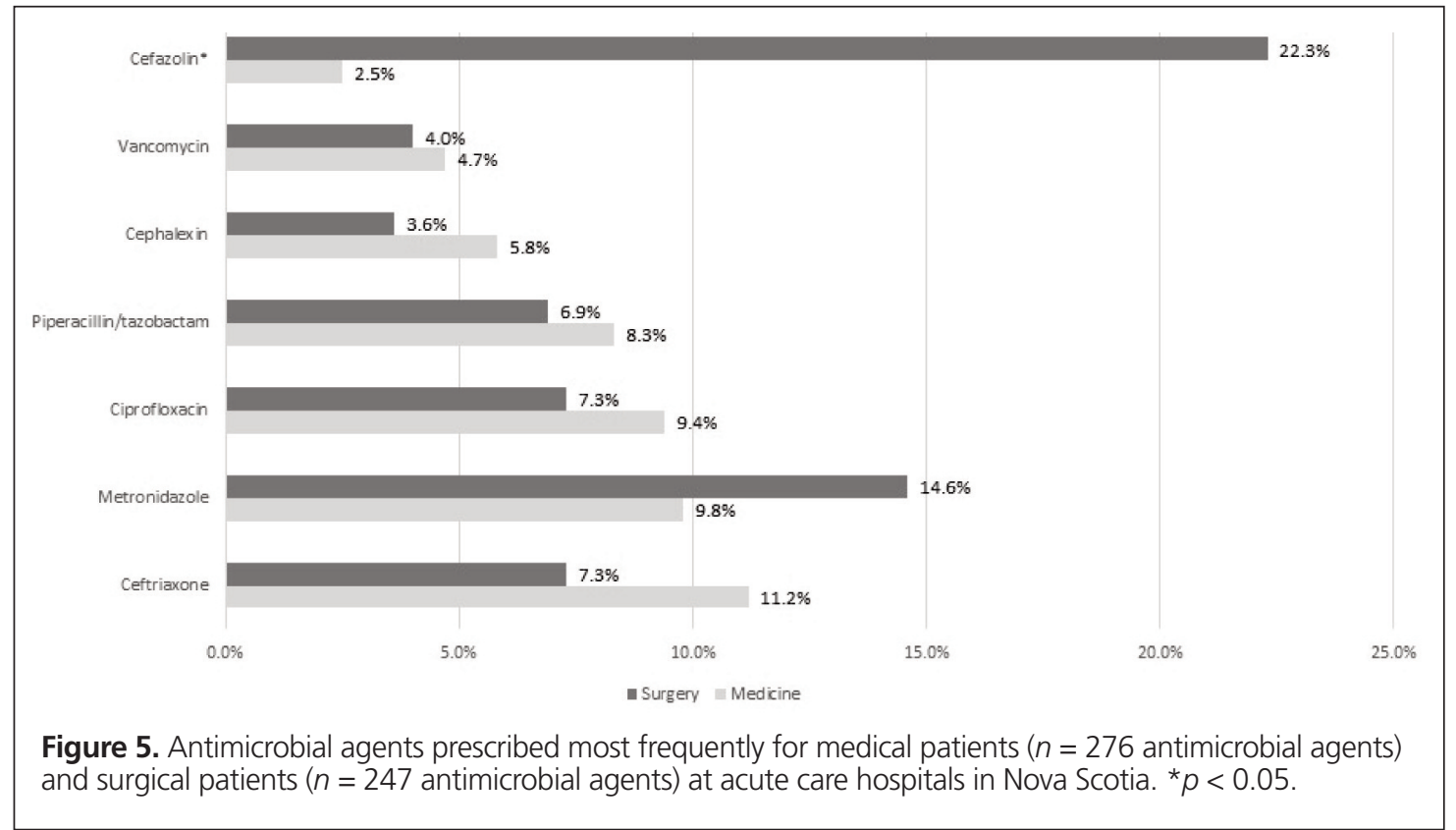

duration of antimicrobial therapy was documented for $37.3 \%$ $(194 / 520)$ of indications.

\section{DISCUSSION}

In this 2015 study, about one-third (30.6\%) of the acute care inpatient population in Nova Scotia received an antimicrobial agent during the study period. These findings are consistent with international and Canadian prevalence data. The most recent transnational point prevalence surveys of antimicrobial use by acute care inpatients in Europe, conducted in 2009 and 2011, reported prevalences of $29.0 \%$ and $34.6 \%$, respectively. ${ }^{9,15} \mathrm{~A}$ point prevalence survey of antimicrobial use in Ontario found that $30.8 \%$ of acute care inpatients were receiving an antimicrobial agent. ${ }^{16}$ Similarly, a point prevalence survey of health careassociated infections at Canadian adult acute care hospitals reported that $36.0 \%$ of patients surveyed were receiving systemic antimicrobial agents. ${ }^{30}$

This study highlights a particularly high prevalence of parenteral antimicrobial use, and conversion from IV to oral therapy was therefore identified as a potential target for quality improvement. Consistent with our findings, frequent and potentially inappropriate use of parenteral antimicrobial agents has been reported elsewhere. ${ }^{9}$ On the basis of findings from the current point prevalence survey and the published literature, the provincial antimicrobial stewardship team in Nova Scotia has developed and is implementing an IV-to-oral conversion protocol to reduce the use of parenteral antimicrobial agents.

Use of broad-spectrum antimicrobial agents should also be considered as a potential area for improvement in antimicrobial utilization, given the level of prescribing of such agents reported here. Particularly concerning was the frequent use of ciprofloxacin for the patients in this study. Consistent with these findings, increasing use of fluoroquinolones, including ciprofloxacin, was previously identified in Nova Scotia. ${ }^{19}$ In addition, use of fluoroquinolones was highlighted as a concern in a point prevalence survey completed at a tertiary care hospital in Ontario. ${ }^{16}$ Ciprofloxacin use represents an important target in small and rural population centres, where limited resources may require prioritization in implementing antimicrobial stewardship programs. Clinicians may benefit from increasing awareness of the risks associated with ciprofloxacin use. In addition, stewardship teams should pay particular attention to ciprofloxacin use in older patients, who are at greater risk from adverse events. ${ }^{31}$

Limited uptake of regional guidelines was also identified as an indicator requiring further attention. Although regional guidelines are disseminated throughout the province, only a third of antimicrobial orders were adherent to these guidelines. Rates of adherence to regional clinical practice guidelines in this study were lower than those described by others. ${ }^{9}$ However, uptake of local guidelines (where available) was more consistent with international findings. ${ }^{9}$ Interpretation of adherence to local guidelines was limited by the small sample size. Further exploration of reasons why prescribers are nonadherent to regional guidelines should be considered by the provincial antimicrobial stewardship team, given that for many conditions, few sites have their own local guidelines. Possible reasons why prescribers do not follow guidelines have been proposed in the literature, including lack of awareness or familiarity, lack of agreement, lack of self-efficacy, lack of outcome expectancy, inertia of previous practice, and external barriers. ${ }^{32}$

A need for improvement in documentation was noted at acute care hospitals in Nova Scotia. For the purposes of this study, full medical charts were reviewed, and approximately 1 in 5 had no documentation of indication, and only a third had documentation of the intended duration of therapy. Consistent with results 
from this study, a large point prevalence survey completed in 25 European countries reported that the indication for antimicrobial prescribing was documented in only $75.7 \%$ of patients' medical charts. ${ }^{9}$ The authors identified documentation (i.e., a statement of the indication in the chart notes) as 1 of the key performance indicators that should be monitored to assess change in targeted areas of practice. ${ }^{9}$ Similarly, a point prevalence survey conducted in Belgium reported that indication for antimicrobial use was documented in only $83.4 \%$ of cases, and the intended duration or date of review was documented in medical records for only $31.9 \%$ of indications. ${ }^{33}$ Documentation improved significantly after an intervention that included education and implementation of a policy requiring prescribers to document indication, name of antibiotic prescribed, and duration or review date in the computerized medical records. After this intervention, the indication was documented in $90.3 \%$ of records and intended duration was listed in $67.7 \%$ of medical records. ${ }^{33}$ To our knowledge, ours is the first Canadian point prevalence survey to formally evaluate documentation.

This study had a number of strengths. To the authors' knowledge, it is the first Canadian point prevalence survey to report use of ESAC-Net methods. Use of these standardized methods allows for international comparison of the findings reported here with the findings of similar surveys completed through ESAC-Net and the ECDC. In addition, this is the first published provincial point prevalence survey in Canada to specifically highlight antimicrobial utilization in small and rural population centres. Researchers considering point prevalence surveys in other parts of Canada will be able to replicate our study using the standardized method that we have reported, which is based on the ESAC-Net protocol for completing point prevalence surveys in large and small population centres.

Although the results of this study provide valuable insight into antimicrobial use by acute care hospitals in Nova Scotia, a number of limitations should be considered. This study was completed as a 1-day survey of antimicrobial use on each participating ward; however, data were not collected on the same day at all participating sites. As a result, seasonal variation may have affected the results. Generalizability to other regions in Canada may also be limited, given that the survey was completed in a single province. Furthermore, pharmacy students and technicians collected some of the data on antimicrobial use, and pharmacy students assessed guideline adherence; all such activities were under the supervision of a pharmacist. To ensure accurate data collection by trainees and the technician, a second member of the research team reviewed data collection for a minimum of $10 \%$ of patient charts and also reviewed all data entry. Assessment of adherence was reviewed by the study investigators (E.B., H.N., K.A., K.S., L.J.). In addition, students participated in educational discussions with the investigators on infectious disease topics, to supplement their course work in the undergraduate pharmacy curriculum. Disagreements in data collection were not documented; however, data collectors subjectively reported few discrepancies. Guideline adherence was assessed for only a subset of the patient population (those with community-acquired pneumonia, acute exacerbation of COPD, UTI, or nonpurulent cellulitis). As a result, the sample size for assessing guideline adherence was limited. Finally, regional guidelines were developed by the tertiary care hospital in Nova Scotia (Queen Elizabeth II Health Sciences Centre) for management of infectious diseases in adults, and these guidelines were made available electronically to all health care providers in the province. However, some prescribers may not have been aware of the availability of guidelines from the tertiary care hospital. Although there are limitations to regional application of guidelines, we felt this was an important question to explore, given the intention of the antimicrobial stewardship team to have a provincial approach.

\section{CONCLUSION}

This study contributes to knowledge about the prevalence of antimicrobial utilization, guideline adherence, and documentation in Canada and can be used locally for benchmarking against internationally published point prevalence data and to identify priorities for antimicrobial stewardship interventions. Key targets for quality improvement that should be prioritized by antimicrobial stewardship teams include conversion from IV to oral route of administration, reduction in the use of broad-spectrum antimicrobials, adherence to guidelines (with particular attention to potentially inappropriate prescribing of ciprofloxacin for UTIs), and improvement in documentation of indication and intended duration of antimicrobial use.

\section{References}

1. Antimicrobial resistance surveillance in Europe 2013. Annual report of the European Antimicrobial Resistance Surveillance Network (EARS-Net). Stockholm (Sweden): European Centre for Disease Prevention and Control; 2014.

2. Zhanel GG, Adam HJ, Baxter MR, Fuller J, Nichol K, Denisuik A, et al. Antimicrobial susceptibility of 22746 pathogens from Canadian hospitals: results of the CANWARD 2007-11 study. J Antimicrob Chemother. 2013;68 Suppl 1:i7-i22.

3. At UN, global leaders commit to act on antimicrobial resistance [news release]. Geneva (Switzerland): World Health Organization; 2016 Sep 21 [cited $2017 \mathrm{Jul} \mathrm{10]}$. Available from: www.who.int/mediacentre/news/ releases/2016/commitment-antimicrobial-resistance/en/

4. Cotta MO, Robertson MS, Upjohn LM, Marshall C, Liew D, Buising KL. Using periodic point-prevalence surveys to assess appropriateness of antimicrobial prescribing in Australian private hospitals. Intern Med J. 2014;44(3): 240-6.

5. Antimicrobial resistance: global report on surveillance 2014. Geneva (Switzerland): World Health Organization; 2017 [cited 2014 Sep 19]. Available from: www.who.int/drugresistance/documents/surveillancereport/en/

6. Tackling antimicrobial resistance and antimicrobial use: a pan-Canadian framework for action. Cat. no. HP40-179/2017E-PDF. Ottawa (ON): Minister of Health; 2017 [cited 2018 Jan 25]. Available from: https://www. canada.ca/en/health-canada/services/publications/drugs-health-products/ tackling-antimicrobial-resistance-use-pan-canadian-framework-action.html

7. Dellit TH, Owens RC, McGowan JE, Gerding D, Weinstein R, Burke J, et al. Infectious Diseases Society of America and the Society for Healthcare Epidemiology of America guidelines for developing an institutional program to enhance antimicrobial stewardship. Clin Infect Dis. 2007;44(2):159-77.

8. Barlam TF, Cosgrove SE, Abbo LM, MacDougall C, Schuetz AN, Septimus EJ, et al. Implementing an antibiotic stewardship program: guidelines by the Infectious Diseases Society of America and the Society for Healthcare Epidmiology of America. Clin Infect Dis. 2016;62(10):e51-e77. 
9. Zarb P, Amadeo B, Muller A, Drapier N, Vankerckhoven V, Davey P, et al. Identification of targets for quality improvement in antimicrobial prescribing: the web-based ESAC Point Prevalence Survey 2009.J Antimicrob Chemother. 2011;66(2):443-9.

10. Seaton RA, Nathwani D, Burton P, McLaughlin C, MacKenzie AR, Dundas $S$, et al. Point prevalence survey of antibiotic use in Scottish hospitals utilising the Glasgow Antimicrobial Audit Tool (GAAT). Int J Antimicrob Agents. 2007;29(6):693-9.

11. Naughton C, Hennessy Y, Mannion C, Philbin M. A comparison of antibiotic point prevalence survey data from four Irish regional/general hospitals. Ir J Med Sci. 2011;180(2):457-61.

12. Amadeo B, Zarb P, Muller A, Drapier N, Vankerckhoven V, Rogues A, et al. European Surveillance of Antibiotic Consumption (ESAC) point prevalence survey 2008: paediatric antimicrobial prescribing in 32 hospitals of 21 European countries. J Antimicrob Chemother. 2010;65(10):2247-52.

13. Ansari F, Erntell M, Goossens H, Davey P. The European Surveillance of Antimicrobial Consumption (ESAC) point-prevalence survey of antibacterial use in 20 European hospitals in 2006. Clin Infect Dis. 2009; 49(10):1496-504.

14. Robert J, Péan Y, Varon E, Bru JP, Bedos JP, Bertrand X, et al. Point prevalence survey of antibiotic use in French hospitals in 2009. J Antimicrob Chemother. 2012;67(4):1020-6.

15. Zarb P, Coignard B, Griskeviciene J, Muller A, Vankerckhoven V, Weist K, et al. The European Centre for Disease Prevention and Control (ECDC) pilot point prevalence survey of healthcare-associated infections and antimicrobial use. Euro Surveill. 2012;17(46):pii 20316.

16. Lee C, Walker SA, Daneman N, Elligsen M, Palmay L, Coburn B, et al. Point prevalence survey of antimicrobial utilization in a Canadian tertiarycare teaching hospital. J Epidemiol Glob Health. 2015;5(2):143-50.

17. Versporten A, Sharland M, Bielicki J, Drapier N, Vankerckhoven V, Goossens $\mathrm{H}$, et al. The antibiotic resistance and prescribing in European children project: a neonatal and pediatric antimicrobial web-based point prevalence survey in 73 hospitals worldwide. Pediatr Infect Dis J. 2013;32(6):e242-53.

18. Blinova E, Lau E, Bitnun A, Cox P, Schwartz S, Atenafu E, et al. Point prevalence survey of antimicrobial utilization in the cardiac and pediatric critical care unit. Pediatr Crit Care Med. 2013;14(6):e280-8.

19. Kent AJ, Sketris IS, Johnston BL, Sommers R. Effect of utilization policies for fluoroquinolones: a pilot study in Nova Scotia hospitals. Can J Hosp Pharm. 2009;62(1):12-20.

20. Antimicrobial use in Canada: antimicrobial use in humans. In: Canadian antimicrobial resistance surveillance system report 2016. Ottawa (ON): Public Health Agency of Canada; 2016 [cited 2017 Jun 2]. Available from: https://www.canada.ca/en/public-health/services/publications/drugs-healthproducts/canadian-antimicrobial-resistance-surveillance-system-report2016.html\#a4-4-1

21. Table 17-10-0005-01: Population estimates on July 1st, by age and sex [data for Nova Scotia]. Ottawa (ON): Statistics Canada; [cited 2018 Jul 26]. Available from: https://www150.statcan.gc.ca/t1/tbl1/en/tv.action?pid= 1710000501\&pickMembers\%5B0\%5D=1.4\&pickMembers\%5B1\%5D=2.1

22. NSHA fact sheet. Halifax (NS): Nova Scotia Health Authority; [cited 2017 Jul 12]. Available from: www.nshealth.ca/nsha-fact-sheet

23. About us. Halifax (NS): IWK Health Centre; [cited 2017 Jul 12]. Available from: www.iwk.nshealth.ca/page/about-us

24. Hospital report: acute care 2007. Ottawa (ON): Ontario Hospital Association and Government of Ontario; 2007 [cited 2017 Jun 2]. Available from: https://secure.cihi.ca/free_products/OHA_Acute07_EN_final_secure.pdf

25. Three missions, one future ... optimizing the performance of Canada's academic health sciences centres. Ottawa (ON): Association of Canadian Academic Healthcare Organizations; 2010 [cited 2017 Jul 12]. Available from: www.healthcarecan.ca/wp-content/themes/camyno/assets/document/ Reports/2010/External/EN/ThreeMissions_EN.pdf

26. Point prevalence survey of healthcare-associated infections and antimicrobial use in European acute care hospitals - protocol version 4.3. Stockholm (Sweden): European Centre for Disease Prevention and Control; 2012.

27. ATC classification index with DDDs, 2015. Oslo (Norway): WHO Collaborating Centre for Drug Statistics Methodology; 2015.
28. Antimicrobial handbook - 2012. Halifax (NS): Capital Health, Department of Pharmacy and Division of Infectious Diseases; 2012.

29. Population centre (POPCTR). Ottawa (ON): Statistics Canada; [modified 2015 Nov 27; cited 2017 Apr 26]. Available from: http://www12. statcan.gc.ca/census-recensement/2011/ref/dict/geo049a-eng.cfm

30. Gravel D, Taylor G, Ofner M, Johnston L, Leob M, Roth VR, et al. Point prevalence survey for healthcare-associated infections within Canadian adult acute-care hospitals. J Hosp Infect. 2007;66(3):243-8.

31. Stahlmann R, Lode H. Safety considerations of fluoroquinolones in the elderly: an update. Drugs Aging. 2010;27(3):193-209.

32. Cabana MD, Rand CS, Powe NR, Wu AW, Wilson MH, Abboud PA, et al. Why don't physicians follow clinical practice guidelines? A framework for improvement. JAMA. 1999;282(15):1458-65.

33. Vercheval C, Gillet M, Maes N, Albert A, Frippiat F, Damas P. Quality of documentation on antibiotic therapy in medical records: evaluation of combined interventions in a teaching hospital by repeated point prevalence survey. Eur J Clin Microbiol Infect Dis. 2016;35(9):1495-500.

Emily Black, BSc(Pharm), ACPR, PharmD, is with Dalhousie University, Halifax, Nova Scotia.

Heather Neville, BSc(Pharm), MSc, is with the Nova Scotia Health Authority, Halifax, Nova Scotia.

Mia Losier, BSc(Pharm), was, at the time this study was conducted, a student at Dalhousie University, Halifax, Nova Scotia. She is now a pharmacy resident with Horizon Health Network, Saint John, New Brunswick.

Megan Harrison, BSC(Pharm), MSc, was, at the time this study was conducted, a student at Dalhousie University, Halifax, Nova Scotia. She is now a staff pharmacist with Horizon Health Network, Saint John, New Brunswick

Kim Abbass, BSc(Pharm), PharmD, is with the Nova Scotia Health Authority, Sydney, Nova Scotia.

Kathy Slayter, BSC(Pharm), PharmD, FCSHP, is with the IWK Health Centre, Halifax, Nova Scotia.

Lynn Johnston, MD, MSC, FRCPC, is with Dalhousie University and the Nova Scotia Health Authority, Halifax, Nova Scotia.

Ingrid Sketris, BSC(Pharm), PharmD, MPA(HSA), FCCP, FCSHP, FCAHS, is with Dalhousie University, Halifax, Nova Scotia.

Competing interests: Lynn Johnston has received grant funding from Viiv-Pfizer for a project unrelated to the work reported here. No other competing interests were declared.

\section{Address correspondence to:}

Dr Emily Black

Dalhousie University, College of Pharmacy

5968 College Street, PO Box 15000

Halifax NS B3H 4R2

\section{e-mail: Emily.Black@dal.ca}

Funding: This study was funded by a Development/Innovative Grant from the Nova Scotia Health Research Foundation and by a Development Grant from the Faculty of Health Professions, Dalhousie University. These grants provided payments to summer research students Mia Losier and Megan Harrison, who performed data collection.

Acknowledgements: The authors would like to acknowledge Joe Fraser for database development and Steve Doucette for statistical support. They would also like to acknowledge Andrea Kent, Nancy McLaughlin, Debbie Davis, Stephanie Lucas, and Angela Dagley-Vaughn for their assistance in facilitating data collection. 